Research Article

\title{
Second-Order Neutral Differential Equations: Improved Criteria for Testing the Oscillation
}

\author{
Osama Moaaz $\mathbb{D}^{1}{ }^{1}$ Ali Muhib $\mathbb{D}^{1,2}$ Saud Owyed, ${ }^{3}$ Emad E. Mahmoud, ${ }^{4,5}$ \\ and Aml Abdelnaser ${ }^{1}$ \\ ${ }^{1}$ Department of Mathematics, Faculty of Science, Mansoura University, Mansoura 35516, Egypt \\ ${ }^{2}$ Department of Mathematics, Faculty of Education-Al-Nadirah, Ibb University, Ibb, Yemen \\ ${ }^{3}$ Department of Mathematics, College of Sciences, University of Bisha, P.O. Box 344, Bisha 61922, Saudi Arabia \\ ${ }^{4}$ Department of Mathematics and Statistics, College of Science, Taif University, P.O. Box 11099, Taif 21944, Saudi Arabia \\ ${ }^{5}$ Department of Mathematics, Faculty of Science, Sohag University, Sohag 82524, Egypt
}

Correspondence should be addressed to Osama Moaaz; o_moaaz@mans.edu.eg

Received 10 October 2020; Revised 12 November 2020; Accepted 16 February 2021; Published 26 February 2021

Academic Editor: Kottakkaran Sooppy Nisar

Copyright (C) 2021 Osama Moaaz et al. This is an open access article distributed under the Creative Commons Attribution License, which permits unrestricted use, distribution, and reproduction in any medium, provided the original work is properly cited.

The main purpose of this study is to establish new improved conditions for testing the oscillation of solutions of second-order neutral differential equation $\left(r(l)\left(u^{\prime}(l)\right)^{\gamma}\right)^{\prime}+q(l) x^{\beta}(\sigma(l))=0$, where $l \geq l_{0}$ and $u(l):=x(l)+p x(\varrho(l))$. By optimizing the commonly used relationship $x>(1-p) u$, we obtain new criteria that give sharper results for oscillation than the previous related results. Moreover, we obtain criteria of an iterative nature. Our new results are illustrated by an example.

\section{Introduction}

In this study, we consider the neutral delay differential equation (NDDE) with second-order of the form

$$
\left(r(l)\left(u^{\prime}(l)\right)^{\gamma}\right)^{\prime}+q(l) x^{\beta}(\sigma(l))=0,
$$

where $l \geq l_{0}$ and $u(l):=x(l)+p x(\varrho(l))$. Throughout the results, we always suppose $\gamma, \beta \in \mathbb{Q}_{\text {odd }}^{+}:=\left\{a / b: a, b \in \mathbb{Z}^{+}\right.$ are odd $\}, \quad p$ is a nonnegative constant, $r \in C\left(\left[l_{0}, \infty\right),[0, \infty)\right), q \in C\left(\left[l_{0}, \infty\right),[0, \infty)\right), \varrho, \sigma \in C\left(\left[l_{0}\right.\right.$, $\infty), \mathbb{R}), q(l)$ is not congruently zero for $l \geq l_{*}$ large enough, $\varrho(l) \leq l, \sigma(l) \leq l, \lim _{l \longrightarrow \infty} \varrho(l)=\lim _{l \longrightarrow \infty} \sigma(l)=\infty$, and

$$
\int_{l_{0}}^{\infty} r^{-(1 / \gamma)}(\xi) \mathrm{d} \xi=\infty .
$$

By a solution of equation (1), we mean a $x \in C^{1}\left(\left[T_{x}, \infty\right)\right)$ for $T_{x} \geq l_{0}$, which has the feature $r\left(u^{\prime}\right)^{\gamma} \in C^{1}\left(\left[T_{x}, \infty\right)\right)$ and satisfies $(1)$ on $\left[T_{x}, \infty\right)$. We only take into account those solutions $x$ that achieve the advantage $\sup \{|x(l)|: l \geq T\}>0$, for all $T \geq T_{x}$. If the solution of (1) is neither ultimately positive nor ultimately negative, then it is called an oscillatory solution; otherwise, it is called nonoscillatory. The equation itself is called oscillatory if all its solutions oscillate.

In real-world life problems, the NDDEs have interesting applications. The NDDEs appear in the modeling of the networks containing lossless transmission lines, in the study of vibrating masses attached to an elastic bar, in the theory of automatic control, and others, see [1]. It is easy-in recent times-to observe the great development in the theory of oscillation for differential equations of different orders.

In the following, we review some of the works that contributed to the development of the oscillation theory of second-order NDDEs were the motivation for this work.

At studying the oscillatory behavior of NDDEs with canonical Case (2), the relationship between the solution and the corresponding function

$$
x(l)>(1-p) u(l)
$$

has been commonly used in the literature. For canonical case (2), by using the Riccati technique, $\mathrm{Xu}$ and Meng [2] 
presented some oscillation criteria for (1) when $\gamma=\beta$. In the case, where $0<p(t) \leq p_{0}<\infty$, Baculikova and Dzurina [3] established the oscillation criteria for (1).

Theorem 1 (see Corollary 2 in [3]). Let $0<\beta \leq 1, \beta \leq \gamma$,

$$
\sigma(l) \leq \varrho(l) \leq l, \varrho^{\prime}(l) \geq \varrho_{0}>0, \varrho \circ \sigma=\sigma \circ \varrho .
$$

$$
\liminf _{l \rightarrow \infty} \int_{\varrho^{-1}(\sigma(l))}^{l} \widehat{G}(s)\left(\int_{l_{1}}^{\sigma(l)} r^{-(1 / \gamma)}(\zeta) \mathrm{d} \zeta\right)^{\beta} \mathrm{d} s>\left(1+\frac{p_{0}^{\beta}}{\varrho_{0}}\right)^{\beta / \gamma} \frac{1}{\mathrm{e}},
$$

then (1) is oscillatory, where $\widehat{G}(l):=\min \{q(l), q(\varrho(l))\}$.

Using relationship (3), Grace et al. [4] studied the oscillatory behavior of solutions of (1) when $\gamma=\beta$ and $p<1$. Moreover, they improved previous results in the literature.

Theorem 2 (see Theorems 3 and 6 in [4]). If

$$
\liminf _{l \rightarrow \infty} \int_{\sigma(l)}^{l} G(s)\left(v^{*}(\sigma(s))\right)^{\gamma} \mathrm{d} s>\frac{1}{\mathrm{e}}
$$

or

$$
\begin{aligned}
& \underset{l \longrightarrow \infty}{\limsup } \int_{l_{1}}^{\infty}\left(\phi(s) \exp \left(-\int_{\sigma(s)}^{s} \frac{\mathrm{d} \zeta}{r^{1 / \gamma}(\zeta) v^{*}(\zeta)}\right)\right. \\
& \left.-\frac{r(s)\left(\phi_{+}^{\prime}(s)\right)^{\gamma+1}}{(\gamma+1)^{\gamma+1} \phi^{\gamma}(s)}\right) \mathrm{d} s=\infty,
\end{aligned}
$$

then (1) is oscillatory, where $v(l):=\int_{l_{1}}^{l} r^{-(1 / \gamma)}(s) d s$, $\phi \in C\left(\left[l_{0}, \infty\right),(0, \infty)\right), \phi_{+}^{\prime}(l):=\max \left\{\phi^{\prime}(l), 0\right\}$, and

$$
\begin{aligned}
G(l) & :=q(l)(1-p(\sigma(l)))^{\gamma}, \\
v_{l_{1}}^{*}(l) & :=v_{l_{1}}(l)+\frac{1}{\gamma} \int_{l_{1}}^{l} v_{l_{1}}(\zeta) v^{\gamma}(\zeta) \mathrm{d} \zeta .
\end{aligned}
$$

Recently, Moaaz et al. [5, 6] generalized and complemented the results in [4]. They established the following criteria for oscillation of (1) with $p<1$.

Theorem 3 (see Theorem 2 in [6]). Let $\beta=\gamma$. If

$$
\liminf _{l \longrightarrow \infty} \frac{\gamma}{\psi(l)} \int_{l}^{\infty} r^{-(1 / \gamma)}(\zeta) \psi^{(\gamma+1) / \gamma}(\zeta) \mathrm{d} \zeta>\frac{\gamma}{(\gamma+1)^{(\gamma+1) / \gamma}}
$$

then (1) is oscillatory, where

$$
\begin{aligned}
& \widehat{v}(l):=\exp \left(-\gamma \int_{\sigma(l)}^{l} \frac{1}{v^{*}(s) r^{(1 / \gamma)}(s)} \mathrm{d} s\right), \\
& \psi(l):=\int_{l}^{\infty} \widehat{v}(\zeta) G(\zeta) \mathrm{d} \zeta
\end{aligned}
$$

and $G(l)$ and $v^{*}(l)$ are defined as in (8).

The similar results as those above have been extended for even-order NDDEs in [7-11]. For the works that dealt with the noncanonical case, that is,

$$
\int_{l_{0}}^{\infty} r^{-(1 / \gamma)}(\xi) \mathrm{d} \xi<\infty
$$

see, for example, [12-14].

The objective of this paper is to establish new oscillation criteria for the NDDE (1) by improving (3). The new relationship enables us to

(i) Create more effective criteria for studying neutral equations in both cases $p<1$ and $p>1$

(ii) Essentially take into account the influence of the delay argument $\varrho(l)$ that has been careless in all related results

(iii) Exclude some restrictions that are usually imposed on the coefficients of the neutral equations in the case where $p>1$

Moreover, we use an iterative technique to establish new oscillation criteria for the $\operatorname{NDDE}(1)$ when $\beta=\gamma$ and $p<1$. One purpose of this paper is to further improve Theorems 2 and 1 . The results reported in this paper generalize, complement, and improve those in [3-6]. To show the importance of our results, we provide an example.

\section{Main Results I: New Relationship between $x$ and $u$}

For simplicity, we just write the functions without the independent variable, such as $f(l):=f$ and $f(g(l))=f(g)$. Moreover, assuming

$$
\begin{aligned}
\varrho^{0} & :=l, \\
\varrho^{m} & :=\varrho \circ \varrho^{m-1}, \\
\varrho^{-m-1} & :=\varrho^{-1} \circ \varrho^{-m}, \quad \text { for } m=1,2, \ldots, \\
\eta_{l_{0}}(l) & :=\int_{l_{0}}^{l} r^{-(1 / \gamma)}(s) \mathrm{d} s, \\
B & := \begin{cases}c_{1}^{\beta-\gamma}, & \text { if } \gamma \leq \beta, \\
c_{2} \eta_{l_{2}}^{\beta-\gamma}(l), & \text { if } \gamma>\beta,\end{cases}
\end{aligned}
$$

where $c_{1}$ and $c_{2}$ are positive constants, the set of all eventually positive solutions of (1) is denoted by $X^{+}$.

Lemma 1 (see Lemma 3 in [3]). Let $x \in X^{+}$. Then,

$$
\begin{aligned}
u & >0, \\
u^{\prime} & >0, \\
\left(r\left(u^{\prime}\right)^{\gamma}\right)^{\prime} & \leq 0,
\end{aligned}
$$

for $l \geq l_{1}$, where $l_{1}$ is sufficiently large. 
The following lemma is a direct observation from the Proof of 2.1 in [5].

Lemma 2. If $x \in X^{+}$, then $u^{\beta-\gamma}(l) \geq B(l)$, eventually.

Lemma 3. Let $x \in X^{+}$and $p>0$, and there exists an even positive integer $n$ such that

$$
\widetilde{p}:=\sum_{m=1}^{n / 2} \frac{1}{p^{2 m-1}}\left(1-\frac{1}{p} \frac{\eta_{l_{2}}\left(\varrho^{-2 m}\right)}{\eta_{l_{2}}\left(\varrho^{-(2 m-1)}\right)}\right)>0 .
$$

Then,

$$
x(l) \geq \widetilde{p}(l) u(l) .
$$

Proof. Suppose that $x \in X^{+}$. Thus, $x(l), x(\varrho(l))$, and $x(\sigma(l))$ are positive for all $l \geq l_{1}$, where $l_{1}$ is sufficiently large. From Lemma 1, we see that (13) holds. Since $\left(r^{1 / \gamma} u^{\prime}\right)^{\prime} \leq 0$, we have that

$$
u(l)>\int_{l_{1}}^{l} \frac{1}{r^{1 / \gamma}(\xi)} r^{1 / \gamma}(\xi) u^{\prime}(\xi) \mathrm{d} \xi>r^{1 / \gamma}(l) u^{\prime}(l) \eta_{l_{1}}(l),
$$

for all $l \geq l_{1}$. Using the definition of $u(l)$, we obtain

$$
x=\frac{1}{p}\left(u\left(\varrho^{-1}\right)-x\left(\varrho^{-1}\right)\right)=\frac{1}{p}\left(u\left(\varrho^{-1}\right)-\frac{1}{p} u\left(\varrho^{-2}\right)\right)+\frac{1}{p^{2}} x\left(\varrho^{-2}\right) .
$$

Repeating this procedure, we obtain

$$
\begin{aligned}
x & =\sum_{m=1}^{n} \frac{(-1)^{m+1}}{p^{m}} u\left(\varrho^{-m}\right)+\frac{1}{p^{n}} x\left(\varrho^{-n}\right) \\
& >\sum_{m=1}^{n / 2} \frac{1}{p^{2 m-1}}\left(u\left(\varrho^{-(2 m-1)}\right)-\frac{1}{p} u\left(\varrho^{-2 m}\right)\right),
\end{aligned}
$$

for $l \geq l_{2} \geq l_{1}$, where $l_{2}$ is sufficiently large, and any even positive integer $n$. Taking (16) and $\varrho^{-2 m} \geq \varrho^{-(2 m-1)}$ into account, we obtain

$$
u\left(\varrho^{-2 m}\right)<u\left(\varrho^{-(2 m-1)}\right) \frac{\eta_{l_{2}}\left(\varrho^{-2 m}\right)}{\eta_{l_{2}}\left(\varrho^{-(2 m-1)}\right)},
$$

for $m=1,2, \ldots,(n / 2)$. Combining (18) and (19), we obtain

$$
\begin{aligned}
& x>\sum_{m=1}^{n / 2} \frac{1}{p^{2 m-1}}\left(1-\frac{1}{p_{0}} \frac{\eta_{l_{2}}\left(\varrho^{-2 m}\right)}{\eta_{l_{2}}\left(\varrho^{-(2 m-1)}\right)}\right) u\left(\varrho^{-(2 m-1)}\right) \\
& >\widetilde{p} u .
\end{aligned}
$$

This completes the proof.

Lemma 4. Let $x \in X^{+}$and $p_{0}<1$. Then,

$$
x(l) \geq \widehat{p}(l) u(l),
$$

for any odd positive integer $n$, where

$$
\widehat{p}:=(1-p) \sum_{m=0}^{(n-1) / 2} p^{2 m} \frac{\eta_{l_{1}}\left(\varrho^{2 m+1}\right)}{\eta_{l_{1}}} .
$$

Proof. Proceeding as in the proof of Lemma 3, we arrive at (16). From the definition of $u(l)$, we have that

$$
x=u-p x(\varrho)=u-p u(\varrho)+p^{2} x\left(\varrho^{2}\right) .
$$

Repeating this procedure, we obtain

$$
\begin{aligned}
x & =\sum_{m=0}^{n}(-1)^{m} p^{m} u\left(\varrho^{m}\right)+p^{n+1} x\left(\varrho^{n+1}\right) \\
& \geq \sum_{m=0}^{(n-1) / 2}\left(p^{2 m} u\left(\varrho^{2 m}\right)-p^{2 m+1} u\left(\varrho^{2 m+1}\right)\right),
\end{aligned}
$$

for $l \geq l_{2} \geq l_{1}$, where $l_{2}$ is sufficiently large, and any odd $n \in \mathbb{Z}^{+}$. Since $\varrho^{2 m+1}(l) \leq \varrho^{2 m}(l)$, we see that

$$
u\left(\varrho^{n}\right) \leq \cdots \leq u\left(\varrho^{2 m+1}\right) \leq u\left(\varrho^{2 m}\right) \leq \cdots \leq u,
$$

for $m=0,2, \ldots,(n-1) / 2$, which with (24) gives

$$
x \geq \sum_{m=0}^{(n-1) / 2} p^{2 m}(1-p) u\left(\varrho^{2 m+1}\right) .
$$

From (16), we find

$$
u\left(\varrho^{2 m+1}\right)>u \frac{\eta_{l_{1}}\left(\varrho^{2 m+1}\right)}{\eta_{l_{1}}},
$$

which with (26) gives

$$
x \geq(1-p) u \sum_{m=0}^{(n-1) / 2} p^{2 m} \frac{\eta_{l_{1}}\left(\varrho^{2 m+1}\right)}{\eta_{l_{1}}} .
$$

This completes the proof.

Theorem 4. Assume that $p_{0}<1$. If there exists a function $\theta \in C^{1}\left(\left[l_{0}, \infty\right),(0, \infty)\right)$ such that

$$
\limsup _{l \rightarrow \infty} \int_{l_{1}}^{l}\left(\theta(\xi) q(\xi) \hat{p}^{\beta}(\sigma(\xi)) \delta(\xi) B(\sigma(\xi))-\frac{1}{(\gamma+1)^{(\gamma+1)}} \frac{r(\xi)\left(\theta_{+}^{\prime}(\xi)\right)^{\gamma+1}}{\theta^{\gamma}(\xi)}\right) \mathrm{d} \xi=\infty
$$


then (1) is oscillatory, where

$$
\begin{aligned}
\hat{\eta}_{l_{0}}(l) & :=\eta_{l_{0}}(l)+\frac{1}{\gamma} \int_{l_{0}}^{l} \eta_{l_{0}}(\xi) \eta_{l_{0}}^{\gamma}(\sigma(\xi)) q(\xi) \hat{p}^{\beta}(\sigma(\xi)) B(\sigma(\xi)) \mathrm{d} \xi \\
\delta(l) & :=\exp \left(-\gamma \int_{\sigma(l)}^{l} \frac{1}{r^{1 / \gamma}(\xi) \widehat{\eta}_{l_{1}}(\xi)} \mathrm{d} \xi\right) .
\end{aligned}
$$

Proof. Assume the contrary that $x$ is a nonoscillatory solution of (1). Without loss of generality, we suppose that $x \in X^{+}$. Thus, $x(l), x(\varrho(l))$, and $x(\sigma(l))$ are positive for all $l \geq l_{1}$, where $l_{1}$ is sufficiently large. Using Lemma 4 , we have that (21) holds. Using (1) and (21), we obtain

$$
\left(r\left(u^{\prime}\right)^{\gamma}\right)^{\prime} \leq-q \widehat{p}^{\beta}(\sigma) u^{\beta}(\sigma) .
$$

Using the chain rule and simple computation, we find

$$
\begin{aligned}
\gamma\left(r^{1 / \gamma} u^{\prime}\right)^{\gamma-1} \frac{\mathrm{d}}{\mathrm{d} l}\left(u-\eta_{l_{1}} r^{1 / \gamma} u^{\prime}\right) & =-\gamma\left(r^{1 / \gamma} u^{\prime}\right)^{\gamma-1} \eta_{l_{1}}\left(r^{1 / \gamma} u^{\prime}\right)^{\prime} \\
& =-\eta_{l_{1}}\left(r\left(u^{\prime}\right)^{\gamma}\right)^{\prime}
\end{aligned}
$$

which with (31) gives

$$
\begin{aligned}
\frac{\mathrm{d}}{\mathrm{d} l}\left(u-\eta_{l_{1}} r^{1 / \gamma} u^{\prime}\right) & \geq \frac{1}{\gamma}\left(r^{1 / \gamma} u^{\prime}\right)^{1-\gamma} \eta_{l_{1}} q \widehat{p}^{\beta}(\sigma) u^{\beta}(\sigma) \\
& \geq \frac{1}{\gamma}\left(r^{1 / \gamma} u^{\prime}\right)^{1-\gamma} \eta_{l_{1}} q \widehat{p}^{\beta}(\sigma) B(\sigma) u^{\gamma}(\sigma) .
\end{aligned}
$$

Integrating (33) from $l_{1}$ to $l$, we obtain

$$
\begin{aligned}
u \geq & \eta_{l_{1}} r^{1 / \gamma} u^{\prime}+\frac{1}{\gamma} \int_{l_{1}}^{l}\left(r^{1 / \gamma}(\xi) u^{\prime}(\xi)\right)^{1-\gamma} \\
& \eta_{l_{1}}(\xi) q(\xi) \widehat{p}^{\beta}(\sigma(\xi)) B(\sigma(\xi)) u^{\gamma}(\sigma(\xi)) \mathrm{d} \xi .
\end{aligned}
$$

Since $\left(\left(r^{1 / \gamma}(l) u^{\prime}(l)\right)^{\gamma}\right)^{\prime} \leq 0$, we have

$$
u(\sigma) \geq \eta_{l_{1}}(\sigma) r^{1 / \gamma}(\sigma) u^{\prime}(\sigma) \geq \eta_{l_{1}}(\sigma) r^{1 / \gamma} u^{\prime}
$$

Thus, (24) becomes

$$
u \geq\left(\eta_{l_{1}}(l)+\frac{1}{\gamma} \int_{l_{1}}^{l} \eta_{l_{0}}(\xi) \eta_{l_{0}}^{\gamma}(\sigma(\xi)) q(\xi) \hat{p}^{\beta}(\sigma(\xi)) B(\sigma(\xi)) \mathrm{d} \xi\right) r^{1 / \gamma} u^{\prime}
$$

that is,

$$
u \geq \widehat{\eta}_{l_{1}} r^{1 / \gamma} u^{\prime}
$$

Integrating $\left(u^{\prime} / u\right) \leq\left(1 /\left(r_{l_{1}}^{1 / \gamma_{l_{1}}}\right)\right)$ from $\sigma(l)$ to $l$, we find

$$
\ln \frac{u(l)}{u(\sigma(l))} \leq \int_{\sigma(l)}^{l} \frac{1}{r^{1 / \gamma}(\xi) \widehat{\eta}_{l_{1}}(\xi)} \mathrm{d} \xi
$$

that is,

$$
u(\sigma(l)) \geq \exp \left(-\int_{\sigma(l)}^{l} \frac{1}{r^{1 / \gamma}(\xi) \widehat{\eta}_{l_{1}}(\xi)} \mathrm{d} \xi\right) u(l)
$$

Next, we define the function

$$
\Theta:=\theta \frac{r\left(u^{\prime}\right)^{\gamma}}{u^{\gamma}} .
$$

Clearly, $\Theta(l)>0$ for all $l \geq l_{1}$ and

$$
\Theta^{\prime}=\frac{\theta^{\prime}}{\theta} \Theta+\theta \frac{\left(r\left(u^{\prime}\right)^{\gamma}\right)^{\prime}}{u^{\gamma}}-\gamma \theta \frac{r\left(u^{\prime}\right)^{\gamma}}{u^{\gamma+1}} u^{\prime} .
$$

It follows from (31) and (39) that

$$
\Theta^{\prime}=\frac{\theta^{\prime}}{\theta} \Theta+-\theta \delta q \widehat{p}^{\beta}(\sigma) B(\sigma)-\gamma \theta \frac{r\left(u^{\prime}\right)^{\gamma}}{u^{\gamma+1}} u^{\prime},
$$

from definition $\Theta$, we have

$$
\Theta^{\prime} \leq \frac{\theta^{\prime}}{\theta} \Theta-\theta \delta q \widehat{p}^{\beta}(\sigma) B(\sigma)-\frac{\gamma}{r^{1 / \gamma} \theta^{1 / \gamma}} \Theta^{1+(1 / \gamma)} .
$$

Using the inequality (see Lemma 1.2 in [5]),

$$
A \phi-B \phi^{(\gamma+1) / \gamma} \leq \frac{\gamma^{\gamma}}{(\gamma+1)^{(\gamma+1)}} \frac{A^{\gamma+1}}{B^{\gamma}}, \quad B>0,
$$

with $A=\left(\theta^{\prime} / \theta\right), B=\left(\gamma /\left(r^{1 / \gamma} \theta^{1 / \gamma}\right)\right)$, and $\phi=\Theta$, we obtain

$$
\Theta^{\prime} \leq-\theta \delta q \widehat{p}^{\beta}(\sigma) B(\sigma)+\frac{1}{(\gamma+1)^{(\gamma+1)}} \frac{r\left(\theta_{+}^{\prime}\right)^{\gamma+1}}{\theta^{\gamma}} \text {. }
$$

Integrating this inequality from $l_{1}$ to $l$, we obtain

$$
\int_{l_{1}}^{l}\left(\theta(\xi) q(\xi) \widehat{p}^{\beta}(\sigma(\xi)) \delta(\xi) B(\sigma(\xi))-\frac{1}{(\gamma+1)^{(\gamma+1)}} \frac{r(\xi)\left(\theta_{+}^{\prime}(\xi)\right)^{\gamma+1}}{\theta^{\gamma}(\xi)}\right) \mathrm{d} \xi \leq \Theta\left(l_{1}\right)
$$


which contradicts (29). This completes the proof.

$$
\limsup _{l \longrightarrow \infty} \int_{l_{1}}^{l}\left(\vartheta(\xi) q(\xi) \tilde{p}^{\beta}(\sigma(\xi)) \widetilde{\delta}(\xi) B(\sigma(\xi))-\frac{1}{(\gamma+1)^{(\gamma+1)}} \frac{r(\xi)\left(\vartheta_{+}^{\prime}(\xi)\right)^{\gamma+1}}{\vartheta^{\gamma}(\xi)}\right) \mathrm{d} \xi=\infty
$$

then (1) is oscillatory, where

$$
\begin{aligned}
& \tilde{\eta}_{l_{0}}(l):=\eta_{l_{0}}(l)+\frac{1}{\gamma} \int_{l_{0}}^{l} \eta_{l_{0}}(\xi) \eta_{l_{0}}^{\gamma}(\sigma(\xi)) q(\xi) \tilde{p}^{\beta}(\sigma(\xi)) B(\sigma(\xi)) \mathrm{d} \xi, \\
& \widetilde{\delta}(l):=\exp \left(-\gamma \int_{\sigma(l)}^{l} \frac{1}{r^{1 / \gamma}(\xi) \tilde{\eta}_{l_{1}}(\xi)} \mathrm{d} \xi\right) .
\end{aligned}
$$

Proof. To prove this theorem, it suffices to use (15) instead of (21) in the proof of Theorem 4.

\section{Main Results II: Iterative Technique}

Lemma 5. Assume that $x \in X^{+}, \gamma=\beta$, and $p_{0}<1$. Then,

$$
u(l) \geq \varphi_{k}(l) r^{1 / \gamma}(l) u^{\prime}(l),
$$

for $k=0,1, \ldots$, where $\varphi_{0}(l):=\widehat{\eta}_{l_{1}}(l)$ and

$$
\varphi_{k+1}(l):=\int_{l_{1}}^{l}\left(\frac{1}{r(s)} \exp \left(\int_{s}^{l} q(\xi) \hat{p}^{\gamma}(\sigma(\xi)) \varphi_{k}^{\gamma}(\sigma(\xi)) \mathrm{d} \xi\right)\right)^{1 / \gamma} \mathrm{d} s .
$$

Proof. Suppose that $x \in X^{+}$. Thus, $x(l), x(\varrho(l))$, and $x(\sigma(l))$ are positive for all $l \geq l_{1}$, where $l_{1}$ is sufficiently large. From Lemma 1, we see that (13) holds. Now, we will prove (49) using induction.

For $k=1$, proceeding as in proof of Theorem 4, we obtain that (31) and (37) hold. From (37), we obtain

$$
u \geq \widehat{\eta}_{l_{1}}(l) r^{1 / \gamma} u^{\prime}=\varphi_{0}(l) r^{1 / \gamma} u^{\prime} .
$$

Next, we assume that (49) holds at $k=n$, that is, $u \geq \varphi_{n} r^{1 / \gamma} u^{\prime}$. Thus, since $\left(\left(r^{1 / \gamma} u^{\prime}\right)^{\gamma}\right)^{\prime} \leq 0$, we find

$$
u(\sigma) \geq \varphi_{n}(\sigma) r^{1 / \gamma}(\sigma) u^{\prime}(\sigma) \geq \varphi_{n}(\sigma) r^{1 / \gamma} u^{\prime} .
$$

which with (31) gives

$$
\left(r\left(u^{\prime}\right)^{\gamma}\right)^{\prime}+q \widehat{p}^{\gamma}(\sigma) \varphi_{n}^{\gamma}(\sigma) r\left(u^{\prime}\right)^{\gamma} \leq 0 .
$$

If we set $H:=r\left(u^{\prime}\right)^{\gamma}$, (53) becomes

$$
H^{\prime}(l)+q \widehat{p}^{\gamma}(\sigma) \varphi_{n}^{\gamma}(\sigma) H(l) \leq 0 .
$$

Applying the Grönwall inequality in (54), we obtain

$$
H(s) \geq H(l) \exp \left(\int_{s}^{l} q(\xi) \widehat{p}^{\gamma}(\sigma(\xi)) \varphi_{n}^{\gamma}(\sigma(\xi)) \mathrm{d} \xi\right),
$$

Theorem 5. Assume that (14) holds for some even positive integer $n$. If there exists a function $\vartheta \in C^{1}\left(\left[l_{0}, \infty\right),(0, \infty)\right)$ such that for $l \geq s \geq l_{1}$, so

$$
u^{\prime}(s) \geq r^{1 / \gamma}(l) u^{\prime}(l)\left(\frac{1}{r(s)} \exp \left(\int_{s}^{l} q(\xi) \hat{p}^{\gamma}(\sigma(\xi)) \varphi_{n}^{\gamma}(\sigma(\xi)) \mathrm{d} \xi\right)\right)^{1 / \gamma} .
$$

Integrating this inequality from $l_{1}$ to $l$, we obtain

$$
\begin{aligned}
u(l) & \geq r^{1 / \gamma}(l) u^{\prime}(l) \int_{l_{1}}^{l}\left(\frac{1}{r(s)} \exp \left(\int_{s}^{l} q(\xi) \bar{p}^{\gamma}(\sigma(\xi)) \varphi_{n}^{\gamma}(\sigma(\xi)) \mathrm{d} \xi\right)\right)^{1 / \gamma} \mathrm{d} s \\
& =\varphi_{n+1}(l) r^{1 / \gamma}(l) u^{\prime}(l) .
\end{aligned}
$$

This completes the proof.

Theorem 6. Assume that $\gamma=\beta$ and $p_{0}<1$. If

$$
\liminf _{l \longrightarrow \infty} \int_{\sigma(l)}^{l} q(\xi) \widehat{p}^{\gamma}(\sigma(\xi)) \varphi_{k}^{\gamma}(\sigma(\xi)) \mathrm{d} \xi>\frac{1}{e},
$$

for some integers $k \geq 0$, then (1) is oscillatory, where $\hat{p}$ and $\varphi_{k}$ are defined as in (22) and (50), respectively.

Proof. Assume the contrary that $x$ is a nonoscillatory solution of (1). Without loss of generality, we suppose that $x \in X^{+}$. Thus, $x(l), x(\varrho(l))$, and $x(\sigma(l))$ are positive for all $l \geq l_{1}$, where $l_{1}$ is sufficiently large. From Lemma 5 , we have that (49) holds. Proceeding as in the proof of Theorem 4, we arrive at (31). Combining (53) and (49), we obtain

$$
\left(r\left(u^{\prime}\right)^{\gamma}\right)^{\prime}(l)+q(l) \widehat{p}^{\gamma}(\sigma(l)) \varphi_{k}^{\gamma}(\sigma(l)) r(\sigma(l))\left(u^{\prime}(\sigma(l))\right)^{\gamma} \leq 0 .
$$

If we set $w:=r\left(u^{\prime}\right)^{\gamma}$, we have that $w$ is a positive solution of the delay differential inequality:

$$
w^{\prime}(l)+q(l) \widehat{p}^{\gamma}(\sigma(l)) \varphi_{k}^{\gamma}(\sigma(l)) w(\sigma(l)) \leq 0 .
$$

Using Theorem 1 in [15], the associated DDE

$$
w^{\prime}(l)+q(l) \hat{p}^{\gamma}(\sigma(l)) \varphi_{k}^{\gamma}(\sigma(l)) w(\sigma(l))=0
$$

has also a positive solution. However, condition (58) ensures oscillation of (61), which is a contradiction. This completes the proof.

Theorem 7. Assume that $\gamma=\beta$ and $p_{0}<1$. If there exists a function $\rho \in C^{1}\left(\left[l_{0}, \infty\right),(0, \infty)\right)$ such that 
TABLE 1: The lower boundaries of the parameter $q_{0}$ in oscillation criteria.

\begin{tabular}{lcccc}
\hline & & $(68)$ & & $(70)$ \\
$(p, \lambda, \mu) \downarrow$ & $n=5$ & $n=9$ & $n=49$ & 5.2474 \\
$((2 / 3), 0.1,0.755)$ & 5.3342 & 5.2529 & 0.8799 & 0.88227 \\
$(0.5,0.5,0.830)$ & 0.8844 & 0.8801 & 1.6857 & 4.41130 \\
$(0.9,0.5,0.900)$ & 2.3491 & 1.9189 & & \\
\hline
\end{tabular}

$$
\limsup _{l \longrightarrow \infty} \int_{l_{1}}^{l}\left(\rho(\xi) q(\xi) \widehat{p}^{\gamma}(\sigma(\xi)) \widehat{\delta}_{k}(\xi)-\frac{1}{(\gamma+1)^{(\gamma+1)}} \frac{r(\xi)\left(\rho_{+}^{\prime}(\xi)\right)^{\gamma+1}}{\rho^{\gamma}(\xi)}\right) \mathrm{d} \xi=\infty
$$

for some integers $k \geq 0$, then (1) is oscillatory, where

$$
\widehat{\delta}_{k}(l):=\exp \left(-\gamma \int_{\sigma(l)}^{l} \frac{1}{r^{1 / \gamma}(\xi) \varphi_{k}(\xi)} \mathrm{d} \xi\right)
$$

and $\widehat{p}$ and $\varphi_{k}$ are defined as in (22) and (50), respectively.

Proof. Assume the contrary that $x$ is a nonoscillatory solution of (1). Without loss of generality, we suppose that $x \in X^{+}$. Thus, $x(l), x(\varrho(l))$, and $x(\sigma(l))$ are positive for all $l \geq l_{1}$, where $l_{1}$ is sufficiently large. Now, we define the function $\psi:=\rho r\left(u^{\prime} / u\right)^{\gamma}$. Thus, $\psi(l)>0$ and

$$
\psi^{\prime}=\frac{\rho^{\prime}}{\rho} \psi+\rho \frac{\left(r\left(u^{\prime}\right)^{\gamma}\right)^{\prime}}{u^{\gamma}}-\gamma \rho r\left(\frac{u^{\prime}}{u^{\gamma+1}}\right)^{\gamma+1} .
$$

From Lemma 5, we have that (49) holds. By replacing (37) with (49) in the proof of Theorem 4, this part of proof is similar to that of Theorem 4 , so we omit it.

Now, we give an example to illustrate our main results.

Example 1. Consider the NDDE:

$$
\left(\left((x(l)+p x(\mu l))^{\prime}\right)^{\gamma}\right)^{\prime}+\frac{q_{0}}{l^{\gamma+1}} x^{\gamma}(\lambda l)=0,
$$

where $q_{0}>0$ and $\mu, \lambda \in(0,1)$. It is easy to verify that $\eta_{l_{0}}(l)=l, \quad \varrho^{m}(l)=\mu^{m} l, \quad \hat{\eta}_{l_{1}}(l)=\left(1+(1 / \gamma) \hat{p}_{0}^{\gamma} q_{0} \lambda^{\gamma}\right) l, \quad$ and $\delta(l)=\lambda^{\gamma}$, where

$$
\begin{aligned}
\widehat{p}(l) & =(1-p) \sum_{m=0}^{(n-1) / 2} p^{2 m} \mu^{2 m+1}:=\widehat{p}_{0}, \\
\widehat{\gamma} & :=\frac{\gamma}{\left(1+(1 / \gamma) \widehat{p}_{0}^{\gamma} q_{0} \lambda^{\gamma}\right)}, \\
\widetilde{p}(l) & =\sum_{m=1}^{n / 2} \frac{1}{p^{2 m-1}}\left(1-\frac{1}{\mu p}\right):=\widetilde{p}_{0}, \\
\widehat{\gamma} & :=\frac{\gamma}{\left(1+(1 / \gamma) \widetilde{p}_{0}^{\gamma} q_{0} \lambda^{\gamma}\right)} .
\end{aligned}
$$
and

Using Theorem 4, we see that (65) is oscillatory if $p<1$

$$
\widehat{p}_{0}^{\gamma} \lambda^{\widehat{\gamma}} q_{0}>\left(\frac{\gamma}{\gamma+1}\right)^{\gamma+1}
$$

Using Theorem 5, we see that (65) is oscillatory if

$$
\tilde{p}_{0}^{\gamma} \lambda^{\gamma} q_{0}>\left(\frac{\gamma}{\gamma+1}\right)^{\gamma+1}
$$

Remark 1. The best-known criteria for oscillation of NDDE (65) are

$$
\begin{gathered}
q_{0}(1-p)^{\gamma} \lambda^{\left(\gamma /\left(1+(1 / \gamma)(1-p)^{\gamma} q_{0} \lambda^{\gamma}\right)\right)} \\
>\left(\frac{\gamma}{\gamma+1}\right)^{\gamma+1}[\text { see [4], Example 3], } \\
q_{0} \lambda^{\gamma} \ln \left(\frac{\mu}{\lambda}\right)>\frac{\mu+p^{\gamma}}{e \mu} \text { [see [3], Corollary 2], }
\end{gathered}
$$

for $p<1$ and $p>1$, respectively.

Giving values for the parameters $p, \mu$, and $\lambda$, we can determine the lower bound of the parameter $q_{0}$ to ensure that every solution of (65) is oscillatory. Table 1 shows the lower boundaries of the parameter $q_{0}$ in different special cases of (65) when $\delta=1$ by using conditions (69) and (67).

Let another particular case of (66), namely,

$$
(x(l)+2 x(\mu l))^{\prime \prime}+\frac{q_{0}}{l^{2}} x\left(\frac{9}{10} l\right)=0 .
$$

Conditions (70) and (68) reduce to $q_{0}>6.2587$ and $q_{0}>1.5881$, respectively.

So, our results improve the related results in $[3,4]$.

Remark 2. Using the boundedness condition $p_{1} \leq p(l) \leq p_{2}$, it will be easy to infer results similar to ours if $p$ is a function in $l$.

\section{Conclusion}

This article is concerned with oscillatory behavior of a class of the neutral delay differential equation (NDDE) with second-order. By optimizing the commonly used relationship $x>(1-p) u$, we obtained new criteria that give sharper results for oscillation than the previous related results. Moreover, we obtained criteria of an iterative nature. Furthermore, in the future work, we can try to get some oscillation criteria of (1) under case of $z(l)=x(l)+c(l) x^{\nu}(\varrho(l)), 0<v<1$. 


\section{Data Availability}

No data were used to support the findings of the study.

\section{Conflicts of Interest}

The authors declare that they have no conflicts of interest.

\section{Authors' Contributions}

The authors contributed equally to this article.

\section{Acknowledgments}

Taif University Researchers Supporting Project number (TURSP-202020), Taif University, Taif, Saudi Arabia.

\section{References}

[1] J. K. Hale, "Functional differential equations," Applied Mathematical Sciences, Vol. 3, Springer-Verlag, New York, NewYork-Heidelberg, 1971.

[2] R. Xu and F. Meng, "Some new oscillation criteria for second order quasi-linear neutral delay differential equations," Applied Mathematics and Computation, vol. 182, no. 1, pp. 797-803, 2006.

[3] B. Baculikova and J. Dzurina, "Oscillation theorems for second-order nonlinear neutral differential equations," Computers and Mathematics with Applications, vol. 2011, no. 62, pp. 4472-4478, 2011.

[4] S. R. Grace, J. Dzurina, I. Jadlovska, and T. Li, “An improved approach for studying oscillation of second-order neutral delay differential equations," Journal of Inequalities and Applications, vol. 2018, no. 193, 2018.

[5] O. Moaaz, "New criteria for oscillation of nonlinear neutral differential equations," Advances in Difference Equations, vol. 2019, no. 484, 2019.

[6] O. Moaaz, M. Anis, D. Baleanu, and A. Muhib, "More effective criteria for oscillation of second-order differential equations with neutral arguments," Mathematics, vol. 8, 986 pages, 2020.

[7] R. P. Agarwal, M. Bohner, T. Li, and C. Zhang, "A new approach in the study of oscillatory behavior of even-order neutral delay differential equations," Applied Mathematics and Computation, vol. 225, pp. 787-794, 2013.

[8] B. Baculikova, J. Dzurina, and T. Li, "Oscillation results for even-order quasilinear neutral functional differential equations," Electronic Journal of Differential Equations, vol. 2011, no. 143, 2011.

[9] T. Li, Z. Han, P. Zhao, and S. Sun, "Oscillation of even-order neutral delay differential equations," Advances in Difference Equations, vol. 2010, 2010.

[10] O. Moaaz, P. Kumam, and O. Bazighifan, "On the oscillatory behavior of a class of fourth-order nonlinear differential equation," Symmetry, vol. 12, no. 4, p. 524, 2020.

[11] O. Moaaz, C. Cesarano, and A. Muhib, "Some new oscillation results for fourth-order neutral differential equations," European Journal of Pure and Applied Mathematics, vol. 13, no. 2, pp. 185-199, 2020.

[12] R. P. Agarwal, C. Zhang, and T. Li, "Some remarks on oscillation of second order neutral differential equations," $A p$ plied Mathematics and Computation, vol. 274, no. 274, pp. 178-181, 2016.

[13] M. Bohner, S. Grace, and I. Jadlovská, "Oscillation criteria for second-order neutral delay differential equations," Electronic
Journal of Qualitative Theory of Differential Equations, vol. 2017, no. 60, pp. 1-12, 2017.

[14] O. Moaaz, E. M. Elabbasy, and B. Qaraad, "An improved approach for studying oscillation of generalized Emden-Fowler neutral differential equation," Journal of Inequalities and Applications, vol. 2020, no. 69, 2020.

[15] C. G. Philos, "On the existence of nonoscillatory solutions tending to zero at ? for differential equations with positive delays," Archiv der Mathematik, vol. 36, no. 1, pp. 168-178, 1981. 